\title{
COLÊMBOLOS (HEXAPODA: COLLEMBOLA) COMO BIOINDICADORES DE QUALIDADE DO SOLO EM ÁREAS COM Araucaria angustifolia(1)
}

\author{
Dilmar Baretta ${ }^{(2)}$, Cristina Seabra Ferreira ${ }^{(3)}$, José Paulo Sousa ${ }^{(4)}$ \\ \& Elke Jurandy Bran Nogueira Cardoso(5)
}

\begin{abstract}
RESUMO
Não existem informações sobre a diversidade de colêmbolos associados às florestas com Araucaria angustifolia (Bert.) O. Kuntze no Brasil. Este estudo teve o objetivo de avaliar o potencial da diversidade de famílias de colêmbolos como bioindicadores da qualidade do solo e para separar diferentes áreas com araucária, utilizando atributos químicos e microbiológicos do solo como variáveis explicativas. O estudo foi realizado em quatro áreas: 1 . floresta nativa com predominância de araucária (NF); 2. reflorestamento de araucária (R); 3. reflorestamento de araucária submetido a incêndio acidental (RF); e 4. pastagem natural com araucárias nativas e ocorrência de incêndio acidental (NPF). Na captura dos organismos, utilizaramse dez armadilhas, distribuídas ao acaso em cada área, nos mesmos pontos de coleta dos atributos químicos e microbiológicos do solo. $\mathrm{O}$ número de colêmbolos de cada família, extraído por armadilhas (abundância), o índice de diversidade de Shannon (H) e a riqueza de famílias foram submetidos à Análise de Variância (ANOVA). A abundância de cada família de Collembola foi submetida à Análise de Componentes Principais (ACP). Considerando as quatro áreas, foram identificadas oito famílias de colêmbolos (Brachystomellidae, Entomobryidae, Hypogastruridae, Isotomidae, Katiannidae, Paronellidae, Sminthuridae e Tomoceridae). A diversidade de famílias de colêmbolos foi maior nas áreas NF e R, em comparação com as áreas RF e NPF. Os atributos químicos e microbiológicos do solo foram
\end{abstract}

(1) Parte da Tese de Doutorado do primeiro autor, apresentada ao Programa de Pós-graduação em Solos e Nutrição de Plantas da Escola Superior de Agricultura Luiz de Queiroz - ESALQ/USP. Trabalho apresentado no XXXI Congresso Brasileiro de Ciência do Solo, 2007 (Gramado, RS).

(2) Professor do Departamento de Zootecnia, Centro de Educação Superior do Oeste - CEO/UDESC. Laboratório de Solos, Rua Benjamin Constant, 84-E, CEP 89806-070, Chapecó (SC). E-mail: dilmarbaretta@gmail.com

(3) Pós-graduanda no Departamento de Zoologia da Faculdade de Ciências e Tecnologia, Universidade de Coimbra. Lg. Marquês de Pombal, 3004-517, Coimbra, Portugal.

(4) Professor do Departamento de Zoologia da Faculdade de Ciências e Tecnologia, Universidade de Coimbra. Lg. Marquês de Pombal, Coimbra, Portugal, 3004-517. E-mail: jps@zoo.uc.pt

(5) Professora Titular do Departamento de Ciência do Solo, Escola Superior de Agricultura "Luiz de Queiroz", Universidade de São Paulo. Av. Pádua Dias, 11, Caixa Postal 9, CEP 13418-900, Piracicaba (SP). E-mail: ejbncard@esalq.usp.br 
eficientes como variáveis explicativas, pois auxiliaram na interpretação das modificações das famílias de colêmbolos. A ACP mostrou que a identificação ao nível de família de Collembola é suficiente para separar as áreas estudadas, além de indicar quais foram as famílias de colêmbolos mais associadas a cada área, o que possibilita sua utilização como bioindicadores de intervenções antrópicas, bem como da qualidade do solo.

Termos de indexação: mesofauna do solo, fauna do solo, atributos microbiológicos do solo, biologia do solo.

\section{SUMMARY: SPRINGTAILS (HEXAPODA: COLLEMBOLA) AS SOIL QUALITY BIOINDICATORS IN AREAS WITHARAUCARIA ANGUSTIFOLIA}

There is no information about the diversity of springtails associated with Araucaria angustifolia (Bert.) O. Kuntze forests in Brazil. This study was carried out to evaluate the potential of springtail family diversity as a soil quality indicator and to separate different Araucaria areas, using soil chemical and microbiological attributes as explanatory variables. The study was conducted in four areas of: 1. native forest with predominance of Araucaria $(N F)$; 2. Araucaria reforestation (R); 3. Araucaria reforestation burnt by an accidental fire (RF); and 4. native grass pasture with native Araucaria and burnt by an intense accidental fire $(\mathrm{NPF})$. In the capture of the organisms, ten Araucaria trees were randomly selected and pitfall traps were distributed around the selected Araucaria trees, at the same sampling points as for soil chemical and microbiological attributes. The number of springtails of each family extracted per trap (abundance), Shannon's diversity index $(H)$ and family richness in the different areas were evaluated by analysis of variance (ANOVA). The abundance of springtails of each family was analyzed by Principal Components Analysis (PCA). Eight springtail families (Brachystomellidae, Entomobryidae, Hypogastruridae, Isotomidae, Katiannidae, Paronellidae, Sminthuridae and Tomoceridae) were identified in the four areas. The diversity of springtail families was always higher in the NF and $R$ areas, in comparison with $R F$ and NPF. The soil chemical and microbiological attributes were effective as explanatory variables, and useful in the interpretation of modifications occurring in springtail families. The PCA showed that the identification at the family level for Collembola was sufficient to separate the four studied areas, and also indicated which springtail families were most associated with each area. This variable can therefore be considered an indicator of soil quality and anthropogenic disturbance in these forests.

Index terms: soil mesofauna, soil fauna, soil microbiological attributes, soil biology.

\section{INTRODUÇÃO}

Araucaria angustifolia (Bert.) O. Kuntze é uma espécie de árvore característica da floresta subtropical brasileira, sendo a principal componente da formação florestal chamada de Floresta Ombrófila Mista (Floresta de Araucária); originalmente ocupava cerca de $200000 \mathrm{~km}^{2}$ e hoje restam apenas 2 a $4 \%$ dessa área (Guerra et al., 2002; Baretta et al., 2007). A crescente intervenção antrópica e o aumento da ocorrência de incêndios acidentais nas áreas com araucária no Estado de São Paulo, provocados direta ou indiretamente pela ação humana, podem contribuir para a extinção dessa planta (classificada como ameaçada) e da diversidade de colêmbolos (Hexapoda: Collembola) a esta associada.
Os colêmbolos são pequenos artrópodes, ápteros, encontrados em todo o mundo (Bellinger et al., 2007). Estão entre os invertebrados mais abundantes no solo, podendo sobreviver também na serapilheira, árvores, litoral marinho e na água doce (Bellinger et al., 2007). Embora estudos com diversidade de colêmbolos no solo tenham aumentado muito nas últimas décadas, no Brasil e no mundo (Sautter \& Santos, 1991; Sautter et al., 1998; Culik \& Zeppelini Fillho, 2003; Ponge et al., 2003; Sousa et al., 2004; Zeppelini Filho \& Bellini, 2004; Cutz-Pool et al., 2007), considerando o crescente interesse na compreensão dos processos ecológicos dos quais esses organismos participam nos ecossistemas, observam-se diversas limitações, tais como: a falta de taxonomistas disponíveis e de métodos adequados para capturar tais organismos (Zeppelini Filho \& Bellini, 2004). 
A utilização dos atributos químicos e microbiológicos do solo como variáveis ambientais explicativas no entendimento do funcionamento do solo, aliada à quantificação da diversidade de invertebrados edáficos como os colêmbolos, é um ponto de partida importante para entender os processos ecológicos de decomposição e ciclagem de nutrientes no solo (Chauvat et al., 2003; Ponge et al., 2003; Cutz-Pool et al., 2007). A diversidade de colêmbolos tem sido usada como bioindicador de intervenções antrópicas, bem como da qualidade do solo (Sautter \& Santos, 1991; Culik et al., 2002; Chauvat et al., 2003; Ponge et al., 2003; Cutz-Pool et al., 2007). Todavia, o conhecimento das interações ecológicas no solo e da diversidade de colêmbolos edáficos na Mata Atlântica é bastante escasso. Desenvolveuse o presente estudo com o objetivo de avaliar, em áreas com araucária naturais e reflorestadas, impactadas ou não pela queima acidental, o potencial da diversidade de famílias de colêmbolos como bioindicadores da qualidade do solo e para separar as áreas, com ênfase na utilização de atributos químicos e microbiológicos do solo como variáveis explicativas.

\section{MATERIAL E MÉTODOS}

O presente trabalho foi realizado no município de Campos do Jordão, SP, em quatro áreas com Araucaria angustifolia (Bert.) O. Kuntze, dentro do Parque Estadual de Campos do Jordão, distante 210 $\mathrm{km}$ da cidade de São Paulo, e situado a $22^{\circ} 39^{\prime}$ latitude Sul e $45^{\circ} 27^{\prime}$ longitude Oeste, com altitude média de $1519 \mathrm{~m}$. O relevo é suave ondulado, com declividade média de $0,1702 \mathrm{~m} \mathrm{~m}^{-1}$. O solo nas áreas estudadas é um Latossolo Vermelho-Amarelo distrófico (Embrapa, 1999). Informações sobre as características químicas do solo podem ser obtidas em Baretta et al. (2007). O clima da região é subtropical de altitude, mesotérmico e úmido (Köppen, Cfb). A maior precipitação pluviométrica concentra-se no verão e no mês de janeiro de 2004, ocorreram mais de $200 \mathrm{~mm}$, enquanto, em setembro do mesmo ano, somente $50 \mathrm{~mm}$; a temperatura média variou de $17,5^{\circ} \mathrm{C}$, em fevereiro de 2004 , a $12^{\circ} \mathrm{C}$, em agosto de 2004 .

As quatro áreas selecionadas foram: 1. floresta nativa com predominância de araucária, em clímax, com baixa interferência antrópica (NF); 2. reflorestamento de araucária, plantado em 1959 (R); 3. reflorestamento de araucária, plantado em 1959, submetido a um incêndio acidental intenso em julho de 2001 (RF) e 4. pastagem natural com araucárias nativas (NPF), submetida a incêndio acidental intenso em setembro de 2004. Todas as áreas (aproximadamente 0,3 ha) estão localizadas em topossequiência e altitudes semelhantes. A área NF é composta basicamente por A. angustifolia e por diversas espécies arbustivas, herbáceas e arbóreas pertencentes às famílias Aquifoliaceae, Araucariaceae, Asteraceae, Cyatheaceae, Lauraceae, Myrsinaceae, Myrtaceae, Podocarpaceae,
Rhamnaceae, Rosaceae, Simaroubaceae, Solanaceae, Symplocaceae, Vochysiaceae e Winteraceae. A área R apresenta maior densidade de araucárias e várias árvores de Podocarpus lambertii Klotz (Podocarpaceae), mas a predominância é das mesmas espécies de araucária da área NF. Por causa do incêndio, a área $\mathrm{RF}$ apresenta menor densidade de araucária (as mesmas espécies de R e NF) e um predomínio da gramínea Aristida longiseta (Poaceae) e da composta Braccharis trimera (Compositae). Maiores informações sobre a flora arbórea, arbustiva e herbácea do Parque Estadual de Campos do Jordão podem ser obtidas em Robim et al. (1990).

A avaliação dos atributos químicos e microbiológicos do solo foi realizada na primeira semana do mês de setembro de 2004 (duas horas após a queima na área NPF). Para tanto, foram escolhidas, ao acaso, dez árvores de araucária por área e, sob a copa de cada uma delas, foram retiradas três subamostras de solo na profundidade de $0-20 \mathrm{~cm}$. O método de amostragem foi padronizado para todos os atributos do solo (coleta no mínimo a $1 \mathrm{~m}$ e no máximo a $2 \mathrm{~m}$ de distância de cada árvore), visando a utilizar todos os atributos edáficos amostrados na análise multivariada. Para as análises, as amostras foram homogeneizadas e passadas em peneira com malha de $2 \mathrm{~mm}$. Uma subamostra foi retirada (em setembro de 2004), para determinação dos seguintes atributos químicos do solo: $\mathrm{pH}$ em $\mathrm{CaCl}_{2}$, matéria orgânica (Método WalkeyBlack), teores de $\mathrm{P}$ (Resina), de $\mathrm{K}$, de $\mathrm{Ca}$ e $\mathrm{Mg}$ trocáveis, conforme método descrito em Raij et al. (2001). O C da biomassa microbiana (CBM) foi determinado pelo método da fumigação-extração (Vance et al., 1987). O C orgânico total (COT) do solo foi estimado por oxidação úmida com dicromato de potássio $\left(\mathrm{K}_{2} \mathrm{Cr}_{2} \mathrm{O}_{4}\right)$ e ácido sulfúrico $\left(\mathrm{H}_{2} \mathrm{SO}_{4}\right)$, conforme proposto por Raij et al. (2001). A partir dos resultados de CBM e COT, foi calculada a relação entre as duas características (Anderson, 1994). A atividade microbiana foi avaliada pela determinação da respiração basal $\left(\mathrm{C}-\mathrm{CO}_{2}\right)$ em $50 \mathrm{~g}$ de amostra de solo (Alef \& Nannipieri, 1995). Utilizaram-se os resultados de $\mathrm{C}-\mathrm{CO}_{2}$ e do CBM para calcular o quociente metabólico $\left(q \mathrm{CO}_{2}\right)$, conforme proposto por Anderson \& Domsch (1993).

A captura dos colêmbolos foi realizada na mesma época da coleta de solo das variáveis explicativas (primeira semana do mês de setembro de 2004). Na captura dos colêmbolos, utilizaram-se armadilhas do tipo "Trampas de Tretzel" (Pitfall traps), constituídas por frascos de vidro com $6 \mathrm{~cm}$ de diâmetro, enterrados no solo com a extremidade vazada nivelada com a superfície do solo, permanecendo no local por três dias (Baretta et al., 2006). Nas armadilhas, foram colocados $200 \mathrm{~mL}$ de solução detergente neutro, na concentração de 2,5\% (Baretta et al., 2007). Dez armadilhas foram instaladas, ao acaso, em cada área, em torno das mesmas árvores de coleta dos atributos químicos e microbiológicos do solo. Após a retirada das armadilhas, as amostras foram passadas em peneiras 
com malhas de $0,20,0,15$ e $0,10 \mathrm{~mm}$, para separar o solo e os fragmentos vegetais, e os animais foram conservados em recipientes com tampa em solução de álcool etílico (75 \%). No laboratório, os colêmbolos foram separados e identificados, com auxilio de literatura específica (Zeppelini Filho \& Bellini, 2004; Bellinger et al., 2007). Como os taxonomistas brasileiros especializados são escassos, os colêmbolos foram identificados até o nível de família, em alguns casos chegando a gênero. Parte das identificações foi realizada na Universidade de Coimbra (Portugal).

A partir do resultado do número de organismos de cada família capturados em cada armadilha (abundância), foram obtidos o índice de diversidade de Shannon (H) e a riqueza de famílias (Odum, 1983; Baretta et al., 2007). Os atributos ecológicos (abundância, $\mathrm{H}$ e riqueza de famílias) foram submetidos à análise de variância (ANOVA), e as médias comparadas por meio do teste de $\operatorname{LSD}(\mathrm{p}<0,05)$, utilizando-se o programa estatístico SAS versão 8.2 (SAS, 2002). A abundância de famílias nas diferentes áreas foi utilizada para a obtenção do comprimento do gradiente (ACD) e, como este foi menor do que três (resposta linear), optou-se pela Análise de Componentes Principais (ACP), usando o programa CANOCO versão 4.0 (Ter Braak \& Smilauer, 1998; Baretta et al., 2007). Os atributos químicos (pH, MO, P, K, Ca e $\mathrm{Mg})$ e microbiológicos do solo $\left(\mathrm{CBM}, \mathrm{C}-\mathrm{CO}_{2}\right.$, relação CBM:COT e $q \mathrm{CO}_{2}$ ) foram utilizados posteriormente na ACP como variáveis explicativas das modificações das famílias de Collembola (Ter Braak \& Smilauer, 1998; Sousa et al., 2004).

\section{RESULTADOS E DISCUSSÃO}

\section{Abundância de famílias de colêmbolos}

Considerando as quatro áreas com araucária, foi extraído um total de 742 colêmbolos, distribuídos em oito famílias (Brachystomellidae, Entomobryidae, Hypogastruridae, Isotomidae, Katiannidae, Paronellidae, Sminthuridae e Tomoceridae). As áreas $\mathrm{NF}$ e $\mathrm{R}$ não apresentaram diferença $(p>0,05)$ na riqueza de famílias de colêmbolos, sendo sete e seis famílias, respectivamente (Quadro 1). A menor riqueza de famílias de colêmbolos foi encontrada nas áreas $\mathrm{RF}$ e NPF, ambas com quatro famílias, sendo essas duas áreas consideradas perturbadas, especialmente por não manter a mesma riqueza da área $\mathrm{NF}$ (Quadro 1). Tendência semelhante foi encontrada para os valores do índice de diversidade de Shannon $(\mathrm{H})$, o qual foi maior na área $\mathrm{NF}(1,73)$, não diferindo entre si as áreas $\mathrm{R}(1,43)$ e $\mathrm{RF}(1,33)$, e menor na $\mathrm{NPF}(0,93)$.

As modificações nos atributos ecológicos pode ser resultado de uma série de fatores, como a heterogeneidade de hábitat, diferenças na riqueza de plantas das áreas,alterações químicas e microbiológicas do solo (Chauvat et al., 2003; Ponge et al., 2003;
Sousa et al., 2004; Cutz-Pool et al., 2007), além de distúrbios provocados pelas intervenções antrópicas que as florestas vêm sofrendo nos últimos anos (Odum et al., 1983; Baretta et al., 2007). As florestas nativas, de maneira geral, apresentam maior heterogeneidade de hábitat e são capazes de suportar maior diversidade de colêmbolos (Chauvat et al., 2003; Sousa et al., 2004).

A presença ou ausência de determinada família de Collembola nas áreas estudadas foi bastante variável, aparentemente não apresentando uma regra lógica (Quadro 1). Nas áreas NPF, RF e NF, a família Entomobryidae apresentou alta abundância, dominando em relação às demais famílias. $\mathrm{Na}$ área $\mathrm{R}$, a família Sminthuridae dominou, mas não foi capturada na área NPF (Figura 1). As demais famílias apresentaram menores valores de abundância nas quatro áreas. Assim, fica evidente que as áreas apresentam grande variabilidade em termos da distribuição da abundância e da diversidade de famílias de colêmbolos. Alguns autores admitem que a diversidade da estrutura da cobertura vegetal pode influenciar a variabilidade da distribuição de comunidades de colêmbolos edáficos (Sautter et al., 1998; Chauvat et al., 2003; Ponge et al., 2003).

A abundância total de colêmbolos foi maior na área NPF (428 colêmbolos) e menor nas áreas NF (115 colêmbolos), RF (103 colêmbolos) e R (96 colêmbolos), não sendo essas três últimas áreas diferentes entre si (Quadro 1). Embora Collembola seja um grupo ubíquo, pouco se sabe sobre a abundância de famílias de colêmbolos no Brasil (Zeppelini Filho \& Bellini, 2004). Alguma precaução deve ser tomada na interpretação dos dados ora apresentados, pois eles se referem somente às famílias de colêmbolos ativas durante o período de estudo. Para trabalhos futuros de diversidade de Collembola, além da utilização de armadilhas, sugere-se o emprego de métodos de amostragens complementares, como a coleta de solo e extração com funil de Berlese-Tullgren, visando à extração de maior diversidade de famílias (Culik et al., 2002; Culik \& Zeppelini Fillho, 2003; Zeppelini Filho \& Bellini, 2004; Cutz-Pool et al., 2007).

\section{Análise de Componentes Principais (ACP)}

$\mathrm{O}$ resultado da ACP demonstrou, por meio da relação entre componente principal 1 (CP 1) e componente principal 2 (CP 2), que houve separação entre as quatro áreas com araucária estudadas. Essa variabilidade dos dados foi explicada em $31,2 \%$ pela CP 1 e $24,0 \%$ pela CP 2, totalizando $55,2 \%$ da variabilidade dos dados de abundância de famílias de colêmbolos (Figura 1). O terceiro componente principal (CP 3) explicou apenas 15,8 \% da variabilidade e foi desconsiderado no presente estudo. Ainda, pôde-se visualizar a maior ou menor associação de cada família de Collembola em cada área com araucária, por meio dos valores absolutos de cada variável (família de Collembola) no lado positivo ou negativo das CP 1 e 
Quadro 1. Abundância de famílias de Collembola ( $\mathrm{n}^{\mathrm{o}}$ total de colêmbolos de cada família) em Florestas de Araucária nativa (NF), introduzida (R), introduzida impactada (RF) e área de pastagem natural com araucárias nativas impactada pela queima acidental $(\mathrm{NPF}) .(\mathrm{n}=10)$

\begin{tabular}{|c|c|c|c|c|}
\hline Família de Collembola & NF & $\mathbf{R}$ & $\mathbf{R F}$ & NPF \\
\hline Hypogastruridae & $\_(1)$ & - & - & - \\
\hline Hypogastrura sp. & 1 & - & - & - \\
\hline Hypogastruridae sp. 1 & 3 & - & - & - \\
\hline Brachystomellidae & - & - & - & - \\
\hline Brachystomella sp. & - & - & - & 4 \\
\hline Isotomidae & - & - & - & - \\
\hline Isotoma $\mathrm{sp}$. & 8 & 4 & 13 & 2 \\
\hline Isotomidae sp. 1 & 4 & - & - & 27 \\
\hline Entomobryidae & - & - & - & - \\
\hline Entomobryidae sp. 1 & 10 & 11 & 1 & 160 \\
\hline Entomobryidae sp. 2 & - & 2 & - & 6 \\
\hline Entomobryidae sp. 3 & 5 & 1 & 23 & - \\
\hline Lepidocyrtus sp. 1 & 11 & 5 & 15 & 224 \\
\hline Lepidocyrtus sp. 2 & 19 & 8 & 24 & 3 \\
\hline Paronellidae & - & - & - & - \\
\hline Paronellidae sp. 1 & 26 & 4 & 10 & - \\
\hline Paronellidae sp. 2 & 1 & - & 1 & - \\
\hline Katiannidae & 10 & 4 & - & - \\
\hline Tomoceridae & 8 & 3 & - & - \\
\hline Sminthuridae & - & - & - & - \\
\hline Sminthuridae sp. 1 & 3 & 34 & 14 & - \\
\hline Sminthuridae sp. 2 & 5 & 5 & 1 & - \\
\hline Sminthuridae sp. 3 & 1 & 15 & - & - \\
\hline Sminthuridae sp. 4 & - & - & 1 & - \\
\hline Abundância de colêmbolos extraídos (D.P.= $=8,94$ ) & $115 \mathrm{~b}$ & $96 \mathrm{~b}$ & $103 \mathrm{~b}$ & $428 \mathrm{a}$ \\
\hline Riqueza de famílias (D.P. $= \pm 1,93$ ) & $7 \mathrm{a}$ & $6 \mathrm{ab}$ & $4 \mathrm{~b}$ & $4 \mathrm{~b}$ \\
\hline Índice de diversidade de Shannon (D.P. $= \pm 0,28$ ) & $1.73 \mathrm{a}$ & $1.43 \mathrm{~b}$ & $1.33 \mathrm{~b}$ & $0.93 \mathrm{c}$ \\
\hline
\end{tabular}

${ }^{(1)}$ : Ausência de colêmbolos. D.P.: Desvio-Padrão. Médias seguidas pela mesma letra, na linha, não diferem entre si pelo teste LSD $(\mathrm{p}<0,05)$.

CP 2. A área $\mathrm{R}$ caracterizou-se por apresentar maior abundância de colêmbolos da família Sminthuridae e baixa abundância das famílias Isotomidae, Brachystomellidae e Entomobryidae, em comparação com as demais áreas estudadas. Já a área RF, comparada às outras áreas, não apresentou forte associação com nenhuma família de Collembola, pois ficou disposta no centro (Figura 1) (valores próximos a zero).

As famílias Paronellidae, Katiannidae e Tomoceridae apresentaram maior associação com a área NF, enquanto as famílias Brachystomellidae,
Isotomidae e Entomobryidae apresentaram associação com a área NPF (Figura 1). A área NF caracterizouse por apresentar maiores valores dos atributos microbiológicos, CBM e C- $\mathrm{CO}_{2}$, e químicos, $\mathrm{MO}, \mathrm{P}$, Ca e $\mathrm{Mg}$, em relação às outras áreas (Figura 1 ), o que pode ter favorecido a maior diversidade de famílias (Cutz-Pool et al., 2007). O CBM não é um componente isolado no solo; ao contrário, interage estreitamente com muitos componentes biológicos (Baretta et al., 2005), inclusive com níveis tróficos mais elevados, como as populações de colêmbolos (Hanlon, 1981; Sousa et al., 2004). A área NPF, ao contrário da área 
$\mathrm{NF}$, apresentou maiores valores de $\mathrm{pH}$ e de $q \mathrm{CO}_{2}$, em comparação às demais áreas. $\mathrm{O}$ valor do $\mathrm{pH}$ do solo tem sido reportado como atributo químico que modifica a comunidade de colêmbolos (Loranger et al., 2001).

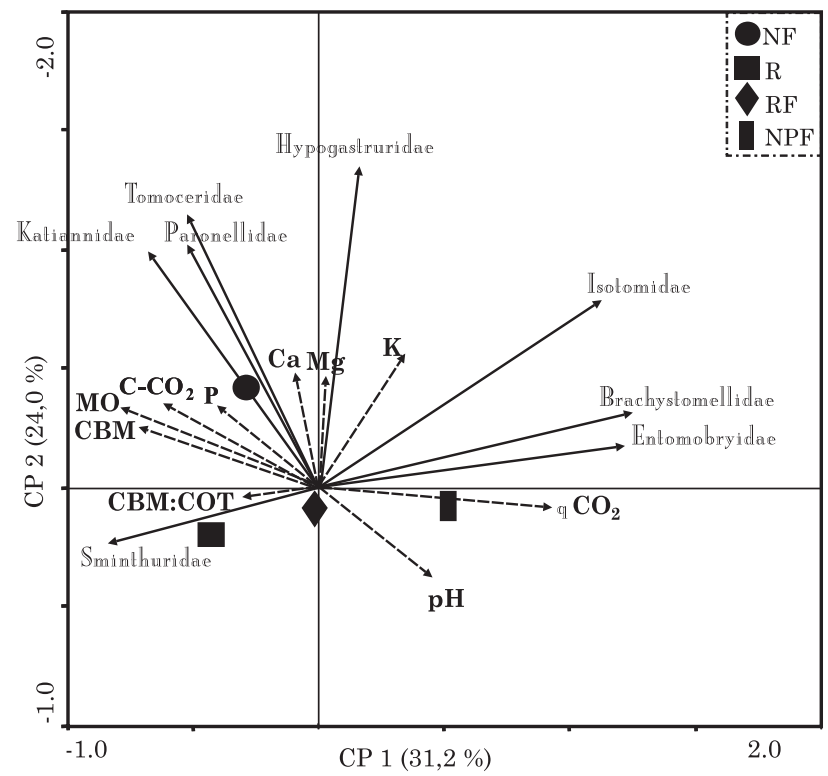

Figura 1. Relação entre o componente principal 1 (CP 1) e o componente principal 2 (CP 2), discriminando Florestas de Araucária nativa (NF: •), introduzida (R: $\square)$, introduzida impactada (RF: $\diamond)$ e área de pastagem natural com araucárias nativas impactada pela queima acidental (NPF: 1), famílias de colêmbolos $(\rightarrow)$ e as variáveis químicas e microbiológicas explicativas $(-\rightarrow)$. pH: Potencial hidrogeniônico; MO: Matéria Orgânica; P: Fósforo; K: Potássio; Ca: Cálcio; Mg: Magnésio; CBM: Carbono da Biomassa Microbiana (CBM); C$\mathrm{CO}_{2}$ : Respiração basal; CBM:COT: relação CBM: Carbono Orgânico Total; $q \mathrm{CO}_{2}$ : Quociente metabólico.

Maiores valores de $q \mathrm{CO}_{2}$ revelaram maiores perdas de $\mathrm{C}$ na área $\mathrm{NPF}$ na forma de $\mathrm{CO}_{2}$ por unidade de $\mathrm{C}$ microbiano (Baretta et al., 2005), enquanto as áreas $\mathrm{NF}, \mathrm{R}$ e RF, ao contrário, apresentaram menores perdas de C. Assim, evidenciou-se que alterações nos atributos químicos e microbiológicos do solo causam modificações na abundância das famílias de colêmbolos, conforme discutido por vários autores (Loranger et al., 2001; Ponge et al., 2003; Sousa et al., 2004; Cutz-Pool et al., 2007).

A partição da variabilidade dos dados pela Análise de Redundância (análise não apresentada) demonstrou que as variáveis ambientais explicativas utilizadas no presente estudo (químicas e microbiológicas do solo), apresentaram grande potencial dentro dos estudos de ecologia do solo, pois explicaram $39,7 \%$ da variabilidade dos dados, sendo $54,5 \%$ desse valor explicado pelo CP 1. Assim, as variáveis ambientais devem ser mais utilizadas como explicativas na ACP, procurando auxiliar na interpretação das interações ecológicas entre as comunidades de Collembola no solo.

A ACP mostrou, além da separação espacial das áreas estudadas, que houve diferenças na distribuição das famílias de colêmbolos (Figura 1), sendo algumas famílias mais sensíveis às interferências antrópicas que as áreas vêm sofrendo nos últimos anos. Dessa forma, a identificação ao nível de família para o grupo Collembola apresenta potencial para ser utilizada como indicador de distúrbios, o que possibilita utilização como bioindicador da qualidade do solo (Ponge et al., 2003; Cutz-Pool et al., 2007). Sabe-se que a maior ou menor sensibilidade de cada família em cada área se deve, dentre outros fatores, às condições climáticas (sazonais), ao tipo de manejo das florestas, histórico das áreas, condições químicas e microbiológicas do solo, além de outros efeitos, como maior quantidade e qualidade dos resíduos vegetais (serapilheira), que proporcionam um ambiente mais ou menos favorável para a sobrevivência das famílias de colêmbolos (Chauvat et al., 2003; Ponge et al., 2003; Cutz-Pool et al., 2007). Essas informações ainda complementam as evidências de que a queima e a intervenção antrópica nas áreas com araucárias podem trazer efeitos negativos drásticos nos atributos químicos e microbiológicos do solo.

\section{CONCLUSÕES}

1. A diversidade e a riqueza de famílias de colêmbolos são sensíveis às intervenções antrópicas em áreas com araucária, o que possibilita sua utilização como bioindicadores de distúrbios, bem como da qualidade do solo.

2. A floresta nativa de araucária proporciona melhores condições edáficas para o desenvolvimento de maior diversidade de famílias de colêmbolos, em comparação às áreas que tiveram maiores interferências antrópicas, especialmente a área de pastagem com queima acidental.

3. A análise de componentes principais (ACP) é importante ferramenta multivariada no estudo de bioindicadores de qualidade do solo, pois permite utilizar atributos químicos e microbiológicos do solo como variáveis ambientais explicativas, o que auxilia na interpretação dos dados ecológicos.

\section{AGRADECIMENTOS}

Os autores agradecem aos integrantes do Laboratório de Solos do Departamento de Zoologia da Faculdade de Ciências e Tecnologia, Universidade de Coimbra (Portugal), e ao Dr. Matthieu Chauvat da 
Universidade de Rouen (França), pela ajuda. Ao Departamento de Ciência do Solo (USP/ESALQ), especialmente aos funcionários Denise Mescolotti, Fernando Baldesin e Dorival Grisotto. Também agradecem ao CNPq, pela bolsa (D. Baretta e E.J.B.N. Cardoso), e ao programa BIOTA/FAPESP (www.biotasp.org.br), pelo financiamento do projeto temático (processo $\mathrm{n}^{\circ}$. 01/05146-6).

\section{LITERATURA CITADA}

ALEF, K. \& NANNIPIERI, P. Methods in applied soil microbiology and biochemistry. London, Academic Press, 1995. $576 \mathrm{p}$

ANDERSON, T.H. \& DOMSCH, K.H. The metobolic quotient for $\mathrm{CO}_{2}\left(q \mathrm{CO}_{2}\right)$ as a specific activity parameter to assess the effects of environment conditions, such as $\mathrm{pH}$, on the microbial biomass of forest soils. Soil Biol. Biochem., 25:393-395, 1993.

ANDERSON, T.H. Physiological analysis of microbial communities in soil: Applications and limitations. In: RITZ, K.D. \& GILLER, K.E., eds. Beyond the biomass. London, British Society of Soil Science, 1994. p.67-76.

BARETTA D.; BRESCOVIT, A.D.; KNYSAK, I. \& CARDOSO, E.J.B.N. Trap and soil monolith sampled edaphic spiders (Arachnida: Araneae) in Araucaria angustifolia forest. Sci. Agric., 64:375-383, 2007.

BARETTA, D.; MAFRA, Á.L.; SANTOS, J.C.P.; AMARANTE, C.V.T. \& BERTOL, I. Análise multivariada da fauna edáfica em diferentes sistemas de preparo e cultivo do solo. Pesq. Agropec. Bras., 41:1675-1679, 2006.

BARETTA, D.; SANTOS, J.C.P.; FIGUEIREDO, S.R. \& KLAUBERG-FILHO, O. Efeito do monocultivo de pinus e da queima do campo nativo em atributos biológicos do solo no Planalto Sul Catarinense. R. Bras. Ci. Solo, 29:715 $724,2005$.

BELLINGER, P.F.; CHRISTIANSEN, K.A. \& JANSSENS, F. Checklist of the Collembola of the world. Disponível em: $<$ http://www.collembola.org $>$. Acesso em: 15 fev. de 2007.

CHAUVAT, M.; ZAITSEV, A.S. \& WOLTERS, V. Successional changes of Collembola and soil microbiota during forest rotation. Oecologia, 137:269-276, 2003.

CULIK, M.P. \& ZEPPELINI FILHO, D. Diversity and distribution of Collembola (Arthropoda: Hexapoda) of Brazil. Biodivers. Conserv., 12: 1119-1143, 2003.

CULIK, M.P.; SOUSA, J.L. \& VENTURA, J.A. Biodiversity of Collembola in tropical agricultural environments of Espírito Santo, Brasil. Appl. Soil Ecol., 21:49-58, 2002.

CUTZ-POOL, L.Q.; PALACIOS-VARGAS, J.G.; CASTAÑOMENESES, G. \& GARCÍA-CALDERÓN, N.E. Edaphic Collembola from two agroecosystems with contrasting irrigation type in Hidalgo State, México. Appl. Soil Ecol., 36:46-52, 2007.
EMPRESA BRASILEIRA DE PESQUISA AGROPECUÁRIA EMBRAPA. Centro Nacional de Pesquisa de Solos. Sistema brasileiro de classificação de solos. Rio de Janeiro, 1999. 412p.

GUERRA, M.P.; SILVEIRA, V.; REIS, M.S. \& SCHNEIDER, L. Exploração, manejo e conservação da araucária (Araucaria angustifolia). In: SIMÕES, L.L. \& LINO, C.F. Sustentável Mata Atlântica: A exploração de seus recursos florestais. São Paulo, SENAC, 2002. p.85-102.

HANLON, R.D. Influences of grazing by Collembola on the activity of senescent fungal colonies on media of different nutrient concentrations. Oikos, 36:362-367, 1981.

LORANGER, G.; BANDYOPADHYAYA, L.; RAZAKA, B. \& PONGE, J.F. Does soil acidity explain altitudinal sequences in Collembolan communities? Soil Biol. Biochem., 33:381393, 2001.

ODUM, E.P. Ecologia. Rio de Janeiro, Guanabara, 1983. 434p.

PONGE, J.F.; GILLET, S.; DUBS, F.; FEDOROFF, E.; HAESE, L.; SOUSA, J.P. \& LAVELLE, P. Collembolan communities as bioindicators of land use intensification. Soil Biol. Biochem., 35:813-826, 2003.

RAIJ, B.van; QUAGGIO, J.A.; CANTARELLA, H. \& ANDRADE, J.C. Análise química para avaliação da fertilidade de solos tropicais. Campinas, Instituto Agronômico de Campinas, 2001. 285p.

ROBIM, M.J.; PASTORE, J.A.; AGUIAR, O.T. \& BAITELLO, J.B. Flora arbóreo arbustiva e herbácea de Parque Estadual de Campos do Jordão (SP). R. Inst. Flor., 2:3153, 1990.

SAS Institute. SAS User's guide: Statistics. Versão 8.2. 6.ed. Cary, 2002.

SAUTTER, K.D. \& SANTOS, H.R. Recuperação de solos degradados pela mineração de xisto, tendo como bioindicadores insetos da Ordem Collembola. Sci. Agr., 11:85-91, 1991.

SAUTTER, K.D.; MOTTA NETO, J.A.; MORAES, A.; SANTOS, H.R. \& RIBEIRO JUNIOR, P.J. População de Oribatei e Collembola em pastagens na recuperação de solos degradados pela mineração do xisto. Pesq. Agropec. Bras., 33:1509-1513, 1998.

SOUSA, J.P.; GAMA, M.M.; PINTO, C.; KEATING, A.; CALHÔA, F.; LEMOS, M.; CASTRO, C.; LUZ, T.; LEITÃO, P. \& DIAS, S. Effects of land-use on Collembola diversity patterns in a Mediterranean landscape. Pedobiologia, 48:609-622, 2004

TER BRAAK, C.J.F. \& SMILAUER, P. CANOCO reference manual and user's guide to Canoco for Windows: Software for canonical community ordination (version 4). New York, Microcomputer Power, 1998.

VANCE, E.D.; BROOKS, P.C. \& JENKINSON, D.S. An extraction method for measuring soil microbial biomass C. Soil Biol. Biochem., 19:703-707, 1987.

ZEPPELINI FILHO, D. \& BELLINI, B.C. Introdução ao estudo dos Collembola. João Pessoa, Universidade Federal da Paraíba, 2004. 82p. 
